\title{
PRODUÇÃO CIENTÍFICA BRASILEIRA SOBRE O NURSING ACTIVITIES SCORE: UMA REVISÃO INTEGRATIVA
}

Tágora do Lago Santos ${ }^{1}$, Lídya Tolstenko Nogueira², Kátia Grillo Padilha ${ }^{3}$

RESUMO: Revisão integrativa de literatura que objetivou identificar o perfil de produções científicas nacionais sobre o Nursing Activities Score e analisar os resultados em evidência de 2002 a 2010. Selecionaram-se 17 artigos na bases de dados Coordenação de Aperfeiçoamento de Pessoal de Nível Superior, Literatura Latino-Americana e do Caribe em Ciências da Saúde; Base de Dados de Enfermagem; Publisher Medline; Scientific Electronic Library Online e Instituto Brasileiro de Informação em Ciência e Tecnologia. Utilizaram-se os termos Nursing Activities Score e Carga de Trabalho de Enfermagem. Encontrou-se nas publicações a identificação da média do Nursing Activities Score, a observação de elementos mais pontuados, a correlação com índices de gravidade e outras ferramentas de dimensionamento de pessoal, como aplicação à clientela idosa, tanto prospectiva como retrospectiva, e, ainda, a criação na forma informatizada. Inferese, portanto, o propósito de viabilizar seu uso como ferramenta prática no gerenciamento em enfermagem.

PALAVRAS-CHAVE: Carga de trabalho; Administração de recursos humanos; Enfermagem; Unidade de terapia intensiva.

\section{BRAZILIAN SCIENTIFIC PRODUCTION ABOUT THE NURSING ACTIVITIES SCORE: AN INTEGRATIVE REVIEW}

ABSTRACT: This integrative revision of the literature aimed to identify the profile of Brazilian scientific productions about the Nursing Activities Score and analyze the results in evidence from 2002 to 2010. 17 articles were elected from the following databases: Coordination for Improvement of Higher Education Personnel (CAPES, in Portuguese), LatinAmerican and Caribbean Literature in Health Sciences; Nursing database; Publisher Medline; Scientific Electronic Library Online and the Brazilian Institute for Information in Science and Technology. The terms Nursing Activities Score and Nursing Workload were used. In the publications, the researchers found the identification of the Nursing Activities Score average, observation of the most heavily-scored elements, the correlation with rates of seriousness and other instruments of dimensioning of personnel, such as application to elderly clients, as much prospective as retrospective, and, further, its creation in informatized form. The goal of viabilizing its use as a practical tool in nursing management is inferred.

KEYWORDS: Workload; Administration of human resources; Nursing; Intensive therapy unit.

\section{PRODUCCIÓN CIENTÍFICA BRASILEÑA SOBRE EL NURSING ACTIVITIES SCORE: UNA REVISIÓN INTEGRATIVA}

RESUMEN: Revisión integrativa de literatura cuyo objetivo fue identificar el perfil de producciones científicas nacionales acerca del Nursing Activities Score y analizar los resultados en evidencia de 2002 a 2010. Fueron seleccionados 17 artículos en las bases de datos Coordinación de Perfeccionamiento de Personal de Nivel Superior, Literatura Latinoamericana y del Caribe en Ciencias de la Salud; Base de datos de Enfermería; Publisher Medline; Scientific Electronic Library Online e Instituto Brasileño de Información en Ciencia y Tecnología. Los términos utilizados fueron Nursing Activities Score y Carga de trabajo de enfermería. En las publicaciones fueron encontradas la identificación de la media del Nursing Activities Score, la observación de elementos más puntuados, la correlación con índices de gravedad y otras herramientas de dimensionamento de personal, como aplicación a la clientela anciana, tanto prospectiva como retrospectiva, y, todavía, la creación en la forma informatizada. Se infiere, por lo tanto, el propósito de volver viable su uso como herramienta práctica en la administración en enfermería. PALABRAS CLAVE: Carga de trabajo; Administración de recursos humanos; Enfermería; Unidad de terapia intensiva.

${ }^{1}$ Enfermeira. Especialista em Terapia Intensiva. Mestranda em Ciências e Saúde pela Universidade Federal do Piauí - UFPI.
${ }^{2}$ Enfermeira. Doutora em Enfermagem pela Universidade Federal do Rio de Janeiro. Professora Associada da UFPI.
${ }^{3}$ Enfermeira. Professora Titular do Departamento de Enfermagem Médico-Cirúrgica da Escola de Enfermagem da Universidade de São Paulo.

Autor correspondente:

Recebido: 29/03/2011

Tágora do Lago Santos

Aprovado: 25/03/2012

Universidade Federal do Piauí

Conjunto Saci, Quadra 37, Casa 20 - 64020-240 -Teresina-PI-Brasil.

E-mail: tagora22@hotmail.com

Cogitare Enferm. 2012 Abr/Jun; 17(2):362-8 


\section{INTRODUÇÃO}

As Unidades de Tratamento Intensivo (UTI) atendem pacientes graves ou de risco que necessitam de assistência médica e de enfermagem de forma ininterrupta, agregando equipamentos tecnológicos específicos e recursos humanos especializados ${ }^{(1)}$.

Os trabalhadores dessas unidades precisam de preparo ao se defrontar com situações que definem o limite entre a vida e a morte das pessoas. A qualidade do cuidado de enfermagem é consequente não somente da qualificação dos trabalhadores, mas, também, da adequada quantificação destes para o desenvolvimento das atividades previstas legalmente; isso contribui para o cuidado de qualidade, possibilitando menor incidência de agravos à saúde dos profissionais e menores riscos à saúde da clientela ${ }^{(2)}$.

Para a alocação adequada de pessoal em UTI faz-se necessária a avaliação da carga de trabalho de enfermagem de acordo com as necessidades de assistência do paciente. Uma equipe superdimensionada se torna mais cara, ao passo que uma equipe reduzida pode provocar menor qualidade/eficácia no atendimento, prolongando a hospitalização e o aumento dos custos do atendimento ao paciente ${ }^{(3)}$.

O termo carga de trabalho incorporou-se aos estudos de dimensionamento de pessoal de enfermagem no sentido de estimar a demanda de trabalho requerida pela equipe. Utilizam-se escores que indicam o número de horas dedicadas pela equipe de enfermagem a cada paciente, considerando-se as peculiaridades e os custos envolvidos em UTI ${ }^{(4)}$. Sendo essas unidades destinadas ao tratamento de pacientes graves, porém recuperáveis, tornou-se imperativo o desenvolvimento de sistemas objetivos de medidas e de métodos prognósticos específicos para identificar quem é o paciente que necessita de cuidados intensivos, e avaliar a real gravidade de suas funções ${ }^{(5)}$. Portanto, os indicadores de carga de enfermagem têm se tornado cada vez mais necessários para garantir a segurança do paciente, para melhorar a qualidade do atendimento e relação custo-efetividade em UTI.

O Nursing Activities Score (NAS) é um instrumento que surgiu na tentativa de adequação quantitativa que expressa a porcentagem de tempo real gasto por um profissional de enfermagem na assistência direta ao doente crítico durante 24 horas ${ }^{(6)}$. Foi traduzido e validado para o português e demonstrou índices satisfatórios de confiabilidade, de validade de critério e de constructo ${ }^{(7)}$. Vêm-se aumentando, lentamente, o número de estudos que utilizam o NAS; a tímida produção pode ser justificada por ser um instrumento novo e recentemente validado e adaptado para a realidade brasileira ${ }^{(8)}$.

A busca de evidências para consolidar o conhecimento tem sido utilizada recentemente na Enfermagem. Consiste na aplicação de pesquisas que embasam a tomada de decisões na assistência à saúde, para tanto, são propostas com este estudo bases que possam subsidiar a pesquisa em Enfermagem ${ }^{(9)}$. Sendo a revisão integrativa de literatura uma via de acesso ao conhecimento já produzido, frente ao tema e o desejo das autoras de partir de resultados e evidências sobre esse, formulou-se a seguinte pergunta: quais são as evidências na literatura produzidas sobre o NAS, no período de 2002 a 2010 ? O objetivo deste estudo foi identificar o perfil das produções e analisar os resultados em evidência sobre o NAS na produção brasileira da enfermagem no período de 2002 a 2010. Pretendeuse contribuir para a orientação quanto à utilização do NAS na prática clinica, sendo possível sua aplicação diária e efeitos diretos sobre o dimensionamento da equipe de enfermagem de acordo, inclusive, com o perfil da clientela; consequentemente, o reflexo dessas mudanças poderá ser observado na assistência de enfermagem.

\section{MÉTODO}

Optou-se pelo método de revisão integrativa de literatura que possibilita que pesquisas concluídas sejam resumidas para se obter conclusões a partir do tema de interesse e que para ser realizada exige os mesmos padrões de clareza, rigor e replicação dos estudos primários ${ }^{(10)}$. Na operacionalização desta revisão seguiram-se estas etapas: seleção da questão temática, estabelecimento dos critérios de seleção da amostra, busca com descritores nos bancos de pesquisa, representação das características da pesquisa original, análise dos dados, interpretação dos resultados e apresentação da revisão ${ }^{(11)}$.

Foram utilizadas as bases de dados da Coordenação de Aperfeiçoamento de Pessoal de Nível Superior (CAPES), Literatura Latino-Americana e do Caribe em Ciências da Saúde (LILACS), Base de Dados de Enfermagem (BDENF), Publisher Medline (PubMed), Scientific Electronic Library Online (SciELO) e Instituto Brasileiro de Informação em Ciência e Tecnologia (IBICT); foram aplicadas as palavras-chave "Nursing Activities Score" e "Carga de Trabalho de Enferma- 
gem". Inicialmente, foram encontradas 35 publicações na CAPES, 23 no LILACS, 10 no BDENF, 790 no PubMed e 21 no SciELO. Seguiu-se com a prévia leitura dos resumos e seleção do período de 2002 a 2010. Para inclusão nesta revisão consideraram-se os trabalhos nos quais o NAS era o instrumento da pesquisa, independente da língua de publicação do periódico e filtraram-se os que se repetiam nas diferentes bases de dados, obtendo-se um total de 20 artigos, 5 dissertações e 4 teses.

Após a leitura das pesquisas, optou-se por considerar para análise somente os artigos, tendo em vista que as dissertações e teses frequentemente foram publicadas nesse formato. Por fim, foram excluídos três artigos que aplicaram o NAS fora do Brasil, para não interferirem no delineamento do perfil nacional.

Realizou-se a avaliação crítica dos artigos selecionados para verificar se respondiam plenamente à pergunta-guia; a amostra final foi composta por 17 artigos. Foi aplicado um formulário de coleta de dados que permitiu a obtenção de informações sobre: identificação do artigo e autores; fonte de localização; objetivos, delineamento e características do estudo; análise dos dados, resultados e discussão, conclusões e recomendações para a prática de Enfermagem. A partir de então se coletaram os dados, com base nas informações que caracterizavam os artigos e, por fim, foram sintetizados e organizados em resultados que permitiram orientar a discussão.

\section{RESULTADOS}

Entre os 17 artigos selecionados, um tratava exclusivamente da tradução e validação do instrumento NAS para a língua portuguesa e foi extraído de uma dissertação de mestrado ${ }^{(7)}$. Os demais, na sua totalidade, tratavam de estudos quantitativos, descritivo-exploratórios, que utilizavam formulários como forma de coleta de informações para o NAS. Predominaram as publicações da Revista da Escola de Enfermagem da USP (23,5\%), em segundo lugar da Acta Paulista de Enfermagem e a Intensive and Critical Care Nursing com 17,6\% cada, seguidas pela Revista Latino-Americana de Enfermagem e Texto \& Contexto Enfermagem, igualmente com 11,7\%, além das revistas Cogitare Enfermagem, Revista Brasileira de Terapia Intensiva e Revista Brasileira de Enfermagem, cada uma com uma produção sobre o tema. Percebeu-se que as publicações estavam distribuídas em periódicos diferentes na área de Enfermagem, com temas gerais e em revistas específicas à terapia intensiva (Tabela 1).

Após o ano de 2007, constatou-se crescimento das publicações do instrumento que havia sido validado em português em 2002, o que significa que foi necessário um intervalo de tempo para o conhecimento e até mesmo divulgação do NAS no meio acadêmico e nas instituições hospitalares (Tabela 1).

Tabela 1 - Distribuição dos estudos disponíveis nas bases de dados relacionados ao NAS, segundo fonte e ano de publicação. Brasil, 2011

\begin{tabular}{|c|c|c|c|c|c|c|c|c|c|c|}
\hline \multirow[t]{2}{*}{ ESTUDOS } & \multicolumn{10}{|c|}{ ANOS } \\
\hline & 2002 & 2003 & 2004 & 2005 & 2006 & 2007 & 2008 & 2009 & 2010 & Total \\
\hline $\begin{array}{l}\text { Rev. esc. enferm. } \\
\text { USP }\end{array}$ & - & - & - & - & - & 2 & 1 & 1 & - & 4 \\
\hline $\begin{array}{l}\text { Acta paul. } \\
\text { enferm. }\end{array}$ & - & - & - & - & 1 & - & 1 & - & 1 & 3 \\
\hline $\begin{array}{l}\text { Rev. latino-am. } \\
\text { enfermagem }\end{array}$ & - & - & - & - & - & - & 1 & 1 & - & 2 \\
\hline $\begin{array}{l}\text { Texto \& contexto } \\
\text { enferm. }\end{array}$ & - & - & - & - & - & - & 1 & 1 & - & 2 \\
\hline $\begin{array}{l}\text { Intensive crit. } \\
\text { care nurs. }\end{array}$ & - & - & - & - & - & 1 & 1 & - & 1 & 3 \\
\hline $\begin{array}{l}\text { Rev. bras. ter. } \\
\text { intensiva }\end{array}$ & - & - & - & - & - & 1 & - & - & - & 1 \\
\hline Cogitare enferm. & - & - & - & - & - & 1 & - & - & - & 1 \\
\hline $\begin{array}{l}\text { Rev. bras. } \\
\text { enfermagem }\end{array}$ & - & - & - & 1 & - & - & - & - & - & 1 \\
\hline Total & - & - & - & 1 & 1 & 5 & 5 & 3 & 2 & 17 \\
\hline
\end{tabular}

Cogitare Enferm. 2012 Abr/Jun; 17(2):362-8 
No que se refere à titulação dos autores, a maioria é docente (37,2\%), e entre os não docentes merecem destaque os mestres $(20,3 \%)$ e os enfermeiros assistentes $(18,6 \%)$ das instituições pesquisadas. Os demais $23,9 \%$ não docentes ficaram distribuídos entre graduandos, mestrandos, doutorandos, doutores, especialistas e médicos assistentes.

Entre esses pesquisadores, 52,5\% procedem da Escola de Enfermagem da Universidade de São Paulo (USP), 27,1\% estão distribuídos entre a Universidade Estadual de São Paulo (UNESP), Universidade Federal do Paraná (UFPR), Universidade de Santo Amaro, Universidade Estadual de Maringá (UEM) e Universidade Estadual de Campinas (UNICAMP), e 20,3\% dos autores não informaram a instituição de sua formação.

A maioria dos estudos foi desenvolvida na região Sudeste, com o Estado de São Paulo se sobressaindo como campo de praticamente todas as produções, excetuandose duas realizadas no Estado do Paraná. A maioria (60\%) das instituições hospitalares nas quais foram coletados os dados das pesquisas eram universitários ou públicas, as demais eram hospitais privados.

Com relação à temática dos artigos, praticamente todos apresentavam como objetivo a verificação da média NAS, exceto dois: um trata da tradução e validação do instrumento e o outro traz uma proposta para aplicação do NAS no Brasil(7,12). Encontrou-se média NAS maior que $50 \%$ em $76,4 \%$ dos artigos, inclusive naqueles que separavam suas amostras por grupos, como idosos e não idosos $(66,4 \%$ e $66,3 \%$, respectivamente) ou em análise do NAS prospectiva e retrospectiva $(59,8 \%$ e $52,7 \%)(8,13-22)$. Dentre estes se destacam três cujo NAS é maior que $70 \%(17,19,21)$.

Dos 15 artigos que aplicam o escore NAS, cinco ressaltam quais são os itens mais pontuados nas suas amostras. Percebe-se que entre os três mais pontuados em cada trabalho se destaca o item três, que se refere à medicação, exceto drogas vasoativas, o qual aparece em todos e que pode ser explicado por ser rotina na internação de pacientes o uso de medicações. Seguem-se ainda outros que se repetem: Investigações laboratoriais; Tarefas administrativas e gerenciais; Posicionamento e mobilização dos pacientes; e Realização de procedimentos de higiene $(8,15,18,20,23)$.

\section{DISCUSSÃO}

Os resultados com NAS maior que $50 \%$ revelam elevada demanda de cuidados de enfermagem, o que sinaliza que um profissional é capaz de cuidar integral- mente de apenas um paciente por turno de trabalho, em especial nas situações em que o escore ultrapassa $70 \%{ }^{(16,19,21)}$. Percebe-se evidente preocupação em correlacionar o NAS com escores de gravidade, como o Acute Physiology and Chronic Health Evaluation (APACHE II) e o Simplified Acute Physiology Score (SAPS II). O APACHE II e o SAPS II são métodos internacionalmente reconhecidos pela comunidade científica que permitem calcular o risco de óbito de um paciente ${ }^{(24-25)}$.

Em um estudo brasileiro foi encontrada correlação positiva entre o NAS e o APACHE II $(\mathrm{R}=0,82)$, e os pacientes que obtiveram valores maiores que a média NAS (> 51\%) na amostra utilizada tiveram maior mortalidade, o que validaria, portanto, a hipótese de que quanto mais grave é o paciente, maior é a sua demanda de trabalho para a equipe de enfermagem ${ }^{(26)}$. Estes resultados diferem com os de outra pesquisa que correlacionou o NAS com o SAPS, que encontrou baixa correlação linear. Os autores observaram que os escores SAPS II e APACHE II utilizam a escala de coma de Glasgow e que seu uso pode superestimar o risco de óbito, mas com certeza não o faz com a carga de trabalho ${ }^{(14)}$.

Em estudo com 214 pacientes, encontrou-se relação estatisticamente significativa entre a média da carga de trabalho NAS, nas primeiras 24 horas, com a condição de saída da UTI $(\mathrm{p}=0,02)$ e o tempo de permanência na unidade. Ou seja, os pacientes que evoluíram para óbito e permaneceram mais de seis dias na UTI necessitaram de maior tempo da assistência de enfermagem. Não houve associação direta entre a gravidade (SAPS II), o sexo, a procedência, o tipo de internação e de tratamento com a demanda de trabalho de enfermagem, e que, por isso, outros fatores preditivos de carga de trabalho devem ser investigados, como, por exemplo, o grau de dependência do paciente ${ }^{(12)}$.

A dependência poderia ser avaliada pelo nível de confusão e agitação psicomotora do paciente crítico, pois, uma vez presentes, determinam acréscimo ao tempo de cuidado de enfermagem. Pacientes inconscientes, imóveis, e com monitorização tecnológica de todas as atividades fisiológicas podem demandar menor tempo da equipe de enfermagem do que pacientes ansiosos, assustados, estressados com o ambiente e que podem comunicar dor e desconforto. Em pesquisa de análise de fatores associados à pontuação NAS obtevese resultados semelhantes, constatando maior média de tempo na UTI e maior mortalidade naqueles com elevados valores $\mathrm{NAS}^{(22)}$. Nesse contexto, pesquisadoras que vivenciam o cuidado a pacientes de alta dependência sentiram a necessidade de testar o NAS como possi- 
bilidade de parâmetro para o dimensionamento em suas unidades assistenciais. Encontraram média NAS de $51,47 \%$ (máxima de 78,90 e mínima de 32,57), com desvio padrão de $\pm 11,17$, portanto, considerando que cada ponto NAS corresponde a 14,4 minutos, cada paciente de alta dependência de enfermagem necessitava de 12,3 horas de assistência nas 24 horas $^{(20)}$.

Os valores são maiores que os preconizados pelo cálculo da Resolução n. 293/2004 do Conselho Federal de Enfermagem que recomenda o acréscimo de 0,5 horas da assistência de enfermagem aos pacientes crônicos, com mais de 60 anos, sem acompanhante, classificados como cuidado intermediário $(5,6+0,5=6,1$ horas) e semi-intensiva $(9,4+0,5=9,9 \text { horas })^{(20)}$.

Em outra pesquisa mensurou-se, por meio do NAS, do Nine Equivalents of Nursing Manpower Score (NEMS) e do Therapeutic Intervention Scoring System-28 (TISS-28), a carga de trabalho de enfermagem em uma unidade de pós-operatório de cirurgia cardíaca, a porcentagem de tempo refletida pelo NAS foi maior quando comparada aos demais. Para a unidade ficou estimada a proporção de profissional de enfermagem/paciente baseada no NAS de 1:1, no NEMS de 0,8: 1 e no TISS-28 de 0,8:1. Os resultados revelaram que $2 / 3$ do tempo de um profissional de enfermagem $(73,7 \%)$ foi gasto no cuidado de um único paciente em um plantão de 24 horas ${ }^{(17)}$. Esse valor é muito próximo do encontrado em outro estudo brasileiro realizado em uma UTI Cardiológica, com média NAS $74,2 \%{ }^{(26)}$.

A aplicação retrospectiva e prospectiva do NAS mostrou homogeneidade. A média e mediana NAS prospectiva foi, respectivamente, $59,8 \%$ e $58,6 \%$, enquanto que a retrospectiva foi $52,7 \%$ e $52,1 \%$. Supõe-se que a pontuação retrospectiva discretamente menor pode estar influenciada por falhas no registro das informações, ou por rotinas impostas pela instituição, como exemplo, a verificação de sinais vitais de $2 / 2$ horas, independente da gravidade do paciente. Escores previamente programados poderiam não ter sido realizados por diversas razões. Itens de concordância prospectiva/retrospectiva fraca ou muito fraca constituem atividades de enfermagem, fisioterapia ou procedimentos médicos, previamente programados e possíveis de não ser realizados. Portanto, não consumiram o tempo previsto na avaliação prospectiva ${ }^{(16)}$.

O NAS prospectivo mostra vantagens para a prática diária em terapia intensiva, pois a distribuição dos profissionais de enfermagem é realizada no início do plantão. Além disso, a aplicação prospectiva busca uma assistência mais completa isenta das interferências da estrutura institucional, que possam dificultar a assistência ideal ${ }^{(16)}$. Idosos com doenças crônicas que evoluem para quadros de desestabilização orgânica levam à necessidade crescente de leitos de UTI. Enfatizando as controvérsias na literatura internacional sobre a idade como preditor isolado para prognóstico na UTI, as elevadas taxas de reinternação e óbito após um ano da alta, e a remota possibilidade de recuperação da capacidade funcional anterior, pesquisa recente questionou até que ponto os investimentos terapêuticos variam em relação à idade e investigou se as necessidades dos cuidados de enfermagem discerniam entre idosos e não idosos, levando em conta as intervenções terapêuticas ${ }^{(21)}$.

Para tal desafio, os autores aplicaram o NAS. Encontraram média de $66,5 \%$, mediana $70,7 \%$ e consideraram os resultados elevados, visto que nessa situação a relação funcionário/paciente é maior que 1:2, o que é o preconizado pelas normas e critérios para aberturas de UTI do Ministério da Saúde. Não houve diferença estatisticamente significativa entre idosos (NAS de $66,4 \%$ ) e não idosos (NAS de 66,3\%) nem no volume na aplicação de recursos terapêuticos nos dois grupos, o que representa o uso de investimentos disponíveis igualitariamente a todos independente da idade ${ }^{(18)}$. Essa observação está de acordo com um estudo realizado no Paraná, que atribui às condições clínicas e de dependência em relação à equipe de enfermagem, o grau de complexidade e a intensidade dos cuidados ao paciente, afirmando não haver influência da idade dos pacientes na amostra ${ }^{(27)}$. Por isso, a discussão é instigada a continuar, no sentido de se pensar nas reais indicações de internação de idosos em UTI em virtude não somente do elevado custo financeiro, físico e emocional para o idoso e sua família, mas, também, devido a aspectos éticos cruciais que ocorrem após a admissão nessa unidade, como quando interromper investimentos diante dos princípios da beneficência, não maleficência, justiça e qualidade de vida ${ }^{(18)}$.

Resultados semelhantes foram encontrados quando se comparou três grupos etários de idosos, um de 60 a 69 anos, outro de 70 a 79 anos e o terceiro de 80 anos e mais, e observou-se valor NAS próximo entre eles $(72,4 \%, 74,3 \%$ e $71,9 \%$, respectivamente). Observando nessa amostra os fatores preditores de alta carga de trabalho, verificou-se que a gravidade e o risco de mortalidade foram elevados na população, o que os autores associaram à grande demanda de cuidados de enfermagem - $70 \%$ do tempo de um profissional ${ }^{(21)}$.

Em consequência do crescimento da aplicação do NAS, percebeu-se dificuldades no preenchimento do 
índice em algumas pesquisas. Numa tentativa de tornar uniforme a aplicação prática do instrumento pelos enfermeiros, surgiu uma proposta que adequa alguns itens de acordo com a realidade das unidades, caso não corresponda ao tempo proposto no índice. Para os itens 1- Monitorização e controles (subitens 1a, 1b, 1c); 4-Procedimentos de higiene (4a, 4b, 4c); 7- Suporte e cuidado aos familiares e pacientes (7a, 7b); e 8- Tarefas administrativas e gerenciais $(8 \mathrm{a}, 8 \mathrm{~b}, 8 \mathrm{c})$, foi orientado transformar em categorias a quantidade de horas, com critérios que determinam o tempo normal, além do normal e muito além do normal para realização das respectivas atividades ${ }^{(12)}$.

Um estudo pioneiro apostou na utilização do NAS na forma de aplicativo informatizado para o gerenciamento da enfermagem em terapia intensiva acreditando que a incorporação de uma tecnologia no planejamento da assistência servirá para o redirecionamento dos cuidados. O instrumento foi elaborado com o objetivo de facilitar e tornar rápida e eficiente a coleta e $\mathrm{o}$ processamento dos dados obtidos através do NAS ${ }^{(13)}$.

Recentemente, aplicou-se o NAS para o dimensionamento de enfermagem associado ao cálculo da Resolução n. 293/2004 do Conselho Federal de Enfermagem que estabelece o Índice de Segurança Técnica (IST) e a proporção de enfermeiros, de acordo com o grau de complexidade da clientela sobre o total de profissionais de enfermagem. Na UTI estudada, encontrou-se uma estimativa de profissionais necessários superior ao quadro real. Verificou-se que o ajuste do NAS às recomendações da resolução contribuem para o dimensionamento adequado da equipe de enfermagem e que isso favorece a qualidade e a segurança da assistência, em especial por considerar, dessa forma, o absenteísmo, embora com um IST empírico ${ }^{(28)}$.

\section{CONSIDERAÇÕES FINAIS}

Considera-se que os artigos científicos que tratam sobre o NAS têm crescido numericamente e obtido maior importância a partir de 2007, porém, a utilização do instrumento e as publicações ainda estão restritas ao centro-sul do país. Nota-se também uma grande abertura da temática em periódicos diferentes da enfermagem, o que facilita a disseminação do conhecimento sobre o instrumento.

A partir da validação e tradução do instrumento as pesquisas se voltaram a diversas formas de exploração, desde a verificação da média NAS, a observação de itens mais pontuados, correlação com índices de gravidade e outras ferramentas de dimen- sionamento de pessoal de enfermagem, aplicação à clientela idosa, tanto prospectiva como retrospectiva. Com o amadurecimento da aplicação surgiu a necessidade de adaptações à realidade brasileira e, também, a recente criação de um instrumento informatizado.

Diante do exposto, sugere-se que sejam realizados estudos com o NAS utilizando amostras maiores e representativas da realidade nacional para consolidar o instrumento como ferramenta de gerenciamento da enfermagem.

\section{REFERÊNCIAS}

1. Ministério da Saúde (BR). Portaria n. 1.071, de 4 de julho de 2005: Política Nacional de Atenção ao Paciente Crítico. Diário Oficial da União, [Internet] 08 jun 2005 [acesso em 25 abr 2010]. Disponível: http://www. sobrati.com.br/ms-politica-critico.htm

2. Inoue KC, Matsuda LM. Dimensionamento da equipe de enfermagem da UTI-adulto de um hospital de ensino. Rev. Eletr. Enf. [Internet] 2009;11(1) [acesso em 10 mar 2011]. Disponível: http://www.fen.ufg.br/revista/v11/n1/ v11n1a07.htm

3. Padilha K, Sousa R, Garcia P, Bento S, Finardi E, Hatarashi R. Nursing workload and staff allocation in an intensive care unit: a pilot study according to Nursing Activities Score (NAS). Intens. Care Nurs. 2011;26(2):108-13.

4. Magalhães AMM, Riboldi CO, Dall'Agnol CM. Planejamento de recursos humanos em enfermagem: desafios para as lideranças. Rev Bras Enferm. 2009;62(4):608-12.

5. Ducci AJ, Padilha KG, Telles SCR, Gutierrez BAO. Gravidade de pacientes e demanda de trabalho de enfermagem em Unidade de Terapia Intensiva: análise evolutiva segundo o TISS-28. Rev Bras Ter Intensiva. 2004;16(1):22-7.

6. Miranda DR, Nap R, De Rijk A, Schaufeli W, Iapichino G. Nursing Activities Score. Crit. care med. 2003;31(2):374-82.

7. Queijo AF, Padilha GK. Nursing Activities Score (NAS): adaptação transcultural e validação para a língua portuguesa. Rev Esc Enferm USP. 2009;43(n. esp):1018-25.

8. Gonçalves LA, Padilha KG. Fatores associados à carga de trabalho de enfermagem em unidade de terapia 
intensiva. Rev Esc Enferm USP. 2007;41(4):645-52.

9. Galvão CM, Sawada NO, Trevisan MA. Revisão sistemática: recurso que proporciona a incorporação das evidências na prática da enfermagem. Rev. Latino-Am. Enfermagem. 2004;12(3):1-9.

10. Beyea SC, Nicoll ELH. Writing an integrative review. AORN J. 1998;67(4):877-80.

11. Ganong LH. Integrative reviews of nursing research. Res. nurs. health. 1987;10(1):1-11.

12. Gonçalves LA, Padilha KG. Nursing Activities Score (NAS): a proposal for practical application in intensive care units. Intens. Care Nurs. 2007;23:355-61.

13. Castro NMC, Dell 'Acqua MCQ, Corrente JE, Zornoff CM, Arantes LF. Aplicativo informatizado com o NAS: instrumento para gerenciamento da assistência em unidade de terapia intensiva. Texto Contexto Enferm. 2009;18(3):577-85.

14. Nogueira LS, Santos MR, Mataloun SE, Moock M. Nursing Activities Score: comparação com o índice APACHE II e a mortalidade em pacientes admitidos em unidade de terapia intensiva. Rev Bras Ter Intensiva. 2007;19(3):327-30.

15. Conishi RMY, Gaidzinski RR. Nursing Activities Score (NAS) como instrumento para medir carga de trabalho de enfermagem em UTI adulto. Rev Esc Enferm USP. 2007;41(3):346-54.

16. Ducci AJ, Padilha KG. Nursing Activities Score: estudo comparativo da aplicação retrospectiva e prospectiva em unidade de terapia intensiva. Acta Paul Enferm. 2008;21(4):581-7.

17. Ducci AJ, Zanei SSV, Whitaker IY. Carga de trabalho de enfermagem para quantificar proporção profissional de enfermagem/paciente em UTI cardiológica. Rev Esc Enferm USP. 2008;42(4):673-80.

18. Ciampone JT, Gonçalves LA, Maia FOM, Padilha KG. Necessidades de cuidados de enfermagem e intervenções terapêuticas em unidades de terapia intensiva: estudo comparativo entre pacientes idosos e não idosos. Acta Paul. Enferm. 2006;19(1):28-35.

19. Gonçalves LA, Garcia PC, Toffoleto MC, Telles SCR, Padilha KG. Necessidades de cuidados de enfermagem em terapia intensiva: evolução diária dos pacientes segundo o Nursing Activities Score (NAS). Rev Bras Enferm. 2006;59(1):56-60.
20. Lima MKF, Tsukamoto R, Fugulin FMT. Aplicação do Nursing Activities Score em pacientes de alta dependência de enfermagem. Texto Contexto Enferm. 2008;17(4):638-46.

21. Sousa CR, Gonçalves LA, Toffoleto MC, Leão K, Padilha KG. Preditores da demanda de trabalho de enfermagem para idosos internados em unidade terapia intensiva. Rev. Latino-Am. Enfermagem. 2008;16(2):218-23.

22. Padilha KG, Sousa RMC, Queijo AF, Mendes AM, Miranda DR. Nursing Activities Score in the intensive care unit: analysis of the related factors. Intens. Care Nurs. 2008;24:197-204.

23. Panuto MR, Guirardello EB. Carga de trabalho de enfermagem em uma unidade de gastroenterologia. Rev. Latino-Am. Enfermagem. 2009;17(6):1009-14.

24. Knaus WA, Draper EA, Wagner DP, Zimmerman JE. APACHE II: a severity of disease classification system. Crit. care med. 1985;13:818-29.

25. Le Gall JR, Lemeshow S, Saulnier F. A new simplified acute physiology score (SAPS II) based on a European/north american multicenter study. JAMA. 1993;270:2957-63.

26. Queijo AF, Padilha K. Nursing Activities Score (NAS): adaptação transcultural e validação para a língua portuguesa. Rev Esc Enferm USP. 2009;43(esp):1018-25.

27. Wolff LDG, Mazur CS, Wiezbick C, Barros CB, Quadros VAS. Dimensionamento de pessoal de enfermagem na unidade semi-intensiva de um hospital universitário de Curitiba. Cogitare enferm. 2007;12(2):171-82.

28. Inoue KC, Matsuda LM. Dimensionamento de pessoal de enfermagem em unidade de terapia intensiva para adultos. Acta Paul. Enferm. 2010;23(3):379-84. 